\title{
Time trends in educational differences in lung and upper aero digestive tract cancer mortality in France between 1990 and 2007.
}

Authors: Kim P. Wagenaar ${ }^{1,2,3}$, Michiel R. de Boer ${ }^{3}$, Danièle Luce ${ }^{1,2}$ and Gwenn Menvielle $e^{1,2}$

${ }^{1}$ Inserm U1018, Center for Epidemiology and Population Health, Occupational and Social Determinants of Health, 16 Av. Paul Vaillant Couturier, 94800, Villejuif, France; ${ }^{2}$ University of Versailles Saint Quentin, UMRS1018, 55 avenue de Paris, 78035 Versailles Cedex, France; ${ }^{3}$ Vrije Universiteit, De Boelelaan 1085, $1081 \mathrm{HV}$, Amsterdam, The Netherlands

Corresponding author: Gwenn Menvielle, INSERM U1018 - CESP, Epidemiology of occupational and social determinants of health, 16 Av. Paul Vaillant Couturier, 94800 Villejuif, France, Phone: +33177747420, Fax: +33177747403, E-mail: Gwenn.Menvielle@inserm.fr 


\section{Abstract}

Purpose: We investigated the magnitude of educational differences in lung and upper aero digestive tract (UADT) cancer mortality in France from 1990 to 2007.

Methods: The analyses were based on census data from a representative sample of the French population. Educational level was used as the indicator for socioeconomic status. Educational differences in mortality from lung and UADT cancer were calculated among people aged 3074 and by birth cohort. Two periods were compared: 1990-1998 and 1999-2007. Mortality rates, hazard ratios and relative indices of inequality (RII) were computed. Results: We found higher lung and UADT cancer mortality among those with less education. Inequalities in male UADT cancer mortality remained stable over time $\left(\mathrm{RII}_{1990-1998}=0.21\right.$ (95\% Confidence Interval $\left.0.15-0.29) ; \quad \mathrm{RII}_{1999-2007}=0.17(0.11-0.26)\right)$ whereas inequalities in lung cancer mortality increased among the younger men $\left(\mathrm{RII}_{1990-1998}=0.48(0.28-0.83) ; \mathrm{RII}_{1999-2007}=0.16\right.$ (0.09-0.31)). Among women, inequalities in lung cancer mortality became apparent during the second period with higher mortality among those with less education. This trend was exclusively driven by the younger women, among whom inequalities reached about the same magnitude as among younger men $\left(\mathrm{RII}_{1999-2007}=0.21(0.08-0.56)\right)$.

Conclusions: UADT cancer mortality rates strongly decreased over time for every educational level. This implies that the burden of health associated with socioeconomic inequalities in UADT cancer mortality decreased substantially. Inequalities in lung cancer mortality are increasing among the younger generation and are expected to increase even more. Differences in magnitude of inequalities among men and women may disappear in the coming decades.

Keywords: lung neoplasms mortality, head and neck neoplasms mortality, education, social class and France. 


\section{Introduction}

In Europe lung cancer is the leading cause of cancer related mortality accounting for one-fifth of cancer related deaths, equal to 342100 deaths a year. Upper aero digestive tract (UADT) cancer, accounts for $3.7 \%$ of all cancer deaths. Higher lung cancer mortality rates are found among men from Southern, Central and Eastern European countries and among women from the Northern European countries [1]. Over the last two decades, lung cancer mortality rates have been declining among men and women in Europe [2] although rates are still increasing among women from Spain, France and the Netherlands [3]. UADT cancer mortality rates among men are highest in Central and Eastern Europe. These mortality rates show a decrease over time which is especially steady in France where extremely high rates were observed in the past. Among women UADT cancer mortality rates have been relatively low for all European countries and stable over time [1, 4].

Lung and UADT cancer mortality has been shown to be related to socioeconomic status. Higher lung cancer mortality rates have been found in low socioeconomic groups among men from the Northern and Southern European countries and among women from the northern European countries. Among women from the Southern European countries such as Spain or Italy, higher rates among higher socioeconomic groups have been observed $[5,6]$. In France, during the 1990s, educational differences were reported in favor of the higher educated men. No differences were observed among women, showing an intermediate position between Northern and Southern European countries [7]. For UADT cancer, higher mortality rates were found among lower educated men in all countries, especially in Slovenia, France and Switzerland and among lower educated women in Switzerland, France and the Nordic countries [7].

Although important changes in mortality rates have been observed during the last decades, literature on trends in socioeconomic inequalities in lung and UADT cancer 
mortality is sparse in Europe. Recent trends in lung cancer mortality in Barcelona, Spain, showed stable inequalities over time for men and women from 1992 to 2003, with higher risk among lower educated men and among higher educated women [8]. In France, inequalities in lung cancer mortality among men increased in the late seventies and stabilized after that until 1996. For UADT cancer, inequalities in mortality increased steeply from 1968 until 1996 [9]. For the period from 1996 onwards, no information is available. In addition, trends in socioeconomic inequalities in lung and UADT cancer mortality among women in France are lacking, as the incidence for these neoplasms was low before the 1990s.

The aim of this study was to compare the educational differences in lung and UADT cancer mortality in France for the periods from 1990 to 1998 and 1999 to 2007. Identifying groups that are at greater risk of dying of lung and UADT cancer can help with the development of effective prevention strategies. 


\section{Methods}

Data

The data were obtained from a representative sample of the French population Echantillon Démographique Permanent (EDP) created by the French National Institute of Statistics (INSEE) containing about $1 \%$ of the population. The sample includes all persons born on any one of four specific calendar dates in any year and is regularly updated to include new subjects with any of these birthdays. Data are updated at each successive census. We excluded people born outside of mainland France because their vital status was not adequately recorded. We studied two periods: 1990-1998 and 1999-2007. Each period started at a census and included all people aged 30-74 at census. People were followed until 31/12/1998 for the first period, 31/12/2007 for the second period, death or their $75^{\text {th }}$ birthday, whichever occurred first.

Causes of death were obtained by linkage with the French national death registry (CepiDc, INSERM). The causes of death were identified for over 99\% of the deceased. The underlying causes of death were classified according to the International Classification of Diseases, Ninth Revision (ICD-9) for the years 1990-1999 and the International Classification of Diseases, Tenth Revision (ICD-10) for the years 2000-2007. The ICD code for lung cancer was 162 in ICD-9 and C33-34 in ICD-10. The ICD code for UADT cancer (lip, oral cavity, pharynx and larynx cancer) was 140-149 and 161 in ICD-9 and C00-14 and C32 in ICD-10 [10]. Self-reported level of education at census was used as the indicator for socioeconomic status and categorized in five groups: 1. no diploma, 2. primary education (6-8 years of education (yrs)), 3. lower secondary or upper secondary vocational education (9-11 yrs), 4. upper secondary education (12 yrs) and 5. tertiary education (more than $12 \mathrm{yrs}$ ). The participants with missing values on education were excluded, $156(0.06 \%)$ in 1990 and 16224 
(5.76\%) in 1999. The analyses included 120307 men and 130980 women in the first period and 127843 men and 137833 women in the second period.

\section{Analyses}

Analyses were performed for men and women separately. Lung and UADT cancer mortality were used as the primary outcomes. Age standardized mortality rates (MR) were computed with direct standardization, using the distribution of person years among the whole sample (including both periods) as standard. Educational differences in mortality were quantified using hazard ratios (HR) computed with Cox regression models, with age as the time scale variable and using no diploma as the reference group. In addition, to estimate the relative inequalities, we computed the relative index of inequality (RII) using Cox regression models. The calculation of the RII is based on a ranked variable for education, which specifies for each educational group the mean proportion of the population with a lower level of education. For example the rank of the lowest educational group is calculated as half the proportion of the population in the lowest educational group. The rank of the second lowest educational group is calculated as the proportion of the population with the lowest education plus half of the proportion of the population with the second lowest education level. The RII is then computed by regressing the mortality on this ranked variable. Thus, the RII expresses inequality in the whole socioeconomic continuum and can be interpreted as the ratio of mortality rates between the two extremes of the educational hierarchy. It deviates further from 1 as the educational inequalities in the study population widen. A RII below 1 indicates a negative association between education and mortality with higher mortality rates among people with less education. A RII above 1 indicates a positive association with higher mortality among those with a higher education. As it takes into account the size and the 
relative position of the different educational groups it is well adapted to compare populations with differing educational distributions $[11,12]$.

Within the two periods, analyses were first conducted for the complete samples and then by birth cohort. For each period, two birth cohorts were created. A priori, we did not have a hypothesis regarding a specific cutoff point, thus the population was divided in approximately two equal groups. In the first period, the younger birth cohort contained people born between 1942 and 1959 and the older birth cohort contained people born before 1942. In the second period the cohorts contained people born between 1951 and 1968 and before 1951 . Analyses were performed using SAS 9.1 and STATA 10.0. 


\section{Results}

The level of education was lower among women than among men in the two periods, but it increased for both men and women between 1990-1998 and 1999-2007 (Table 1). The proportion of the population without a diploma decreased from $22 \%$ to $16 \%$ for men and from $25 \%$ to $17 \%$ for women. Conversely, the population with lower or upper secondary vocational education and tertiary education increased. The proportion of people with upper secondary education remained stable over time. The same trend and pattern was observed within the two birth cohorts. In general, the educational level was lower in the older birth cohort.

Men

Among men we found clear negative associations between education and mortality for both periods, which were more pronounced for UADT than for lung cancer mortality (Table 2). When compared with men with no education, the risk of dying from UADT cancer decreased by $27 \%(\mathrm{HR}=0.73(95 \%$ Confidence Interval 0.61-0.89) $)$ among men with primary education and by $88 \%(\mathrm{HR}=0.12(0.07-0.21))$ among men with tertiary education in the first period. The RII for UADT cancer mortality remained stable over time $\left(\mathrm{RII}_{1990-}\right.$ $\left.{ }_{1998}=0.21(0.15-0.29) ; \mathrm{RII}_{1999-2007}=0.17(0.11-0.26)\right)$. Lung cancer showed a slightly different pattern as the association between education and lung cancer mortality was clearer in the second period. The RII decreased from $0.49(0.39-0.61)$ in the first period to $0.41(0.32-0.52)$ in the second period, but this change was not statistically significant $(\mathrm{p}=0.28)$.

Furthermore, inequalities in lung cancer mortality increased over time from 0.48 $(0.28-0.83)$ to $0.16(0.09-0.31)$ among the younger men $(\mathrm{p}=0.01)$, but not for the older men (Table 3). 


\section{Women}

Among women, there was no clear association between lung cancer mortality and education, in the first period (Table 2). In the second period, an association was found with a reduced risk among women with tertiary education $(\mathrm{HR}=0.47(0.28-0.81))$ when compared to women without education. In addition a change in RIIs was observed for lung cancer mortality between the two periods from $1.20(0.65-2.22)$ to $0.67(0.41-1.09)$, although not statistically significant $(\mathrm{p}=0.15)$ (Table 2 ). For UADT cancer, a negative association was suggested in both periods.

No educational differences were observed in lung cancer mortality among older women in both periods. In contrast, the RIIs for younger women changed from $0.60(0.18$ 2.05) in the first period to $0.21(0.08-0.56)$ in the second period, showing a clear negative association in the second period. However, this change in educational inequalities did not reach statistical significance $(\mathrm{p}=0.19)$ (Table 3$)$. In both periods, the RIIs for UADT cancer mortality were similar in the two birth cohorts $\left(\mathrm{RII}_{1990-1998 \text {, younger cohort }}=0.34\right.$ (0.06-1.83); $\mathrm{RII}_{1999-2007 \text { younger cohort }}=0.41(0.08-2.15)$ and $\mathrm{RII}_{1990-1998 \text { older cohort }}=0.39(0.10-1.53) ; \mathrm{RII}_{1999-2007}$ older cohort $=0.45(0.16-1.27))$. 


\section{Discussion}

This study focused on the magnitude of relative educational differences in lung and UADT cancer mortality in France between 1990-1998 and 1999-2007. Different results among men and women were found. For men lung and UADT cancer mortality were negatively associated with education. Inequalities in lung and UADT cancer mortality were stable over time among men aged 30-74. However, inequalities for lung cancer mortality among younger men increased. For women no clear association between education and lung cancer mortality was observed in the first period. In the second period a negative association was found. These inequalities were restricted to the younger women. No inequalities were reported in the older women in both periods. In addition, UADT cancer mortality showed higher rates among less educated women.

Educational differences in smoking consumption account for a large part of educational differences in lung cancer mortality $[13,14]$. Thus the inequalities we found in lung cancer mortality can be partly explained by socioeconomic differences in smoking during the smoking epidemic.

The habit of smoking usually spreads through a population in four stages. During stage 1 smoking is mainly a habit of men with a higher socioeconomic status. In stage 2 smoking becomes more common. The rates among men peak and become equal among the different socioeconomic groups. Women with a higher education start smoking as the spread of smoking among women lags 10-20 years behind men. During stage 3 smoking rates decline among men, especially among those with a higher socioeconomic status. Women reach their peak rate. Throughout stage 4 the smoking rates keep on declining among men and start to decline among women. Smoking becomes more a habit of people with a lower socioeconomic 
status. In this stage the mortality rates, due to smoking, among men peak after one decade. Among women mortality rates peak after two or three decades [15].

According to our results, France is at the beginning of the fourth stage in the smoking epidemic. Lung cancer mortality rates among men seem to have reached their peak, whereas among women they are still increasing which is in accordance with a study on lung cancer mortality trends in Europe [2]. Moreover educational differences in lung cancer mortality are increasing among younger men and are becoming apparent in the second period among women. This is in line with the notion that smoking in this stage becomes more a habit of people with a low socioeconomic status both in men and women.

Our results are also consistent with the pattern of smoking in France. After the Second World War and onwards smoking rates among men were high, whereas women only started to smoke in large numbers in the late 1960s. From 1980 to 2000 first stable and then decreasing smoking rates have been observed among men, while among women smoking rates have increased [16]. This is consistent with the stable lung cancer mortality rates we found among men and the increasing mortality rates we found among women in the 2000s. In addition, a study reported that around 1990 smoking became more prevalent among both men and younger women with less education in France [17]. This is in line with the inequalities in lung cancer mortality we found in the second period.

The results among younger women are particularly striking. The change in inequalities in lung cancer mortality is extremely quick. A strong negative association between education and lung cancer mortality was shown in the second period, whereas in the first period no clear association was observed. These younger women started to smoke in the late 1960s when 
female smoking rates strongly increased. It therefore seems that the massive uptake of smoking in the female population occurred when inequalities in smoking reversed in favor of women with a higher educational level. The inequalities in lung cancer mortality were as large among younger women as among younger men in the second period. To reach this magnitude, men needed several decades, while women only needed one decade. Thus French women seem to go through the phases of the smoking epidemic much more quickly than French men. This result is remarkable, and additional studies are needed to understand the reasons for this quick change in smoking behavior.

Literature on trends in socioeconomic inequalities in lung cancer mortality over time is sparse. The stable educational differences we found among men were also observed in a study conducted in Barcelona investigating the trends from 1992-2003 [8]. However, the increasing negative association which we found among women was not observed. In Barcelona a positive association was reported. Several explanations could be suggested. We studied a more recent time period and therefore the negative association in the latest time period shown in our study may not yet have been observed in Barcelona in 2002. Furthermore there is a north-south gradient in Europe regarding socioeconomic inequalities in lung cancer mortality, as a consequence of the geographical spread of the smoking epidemic [5, 6]. The northern part of Europe is in an advanced stage of the smoking epidemic compared to the southern part of Europe $[5,6,17]$. France lies in the middle [7, 17]. Our results suggest that France is now more similar to northern European countries [7] or the US [18], at least among the younger generation. The reversal and widening of inequalities in lung cancer mortality we observed were also observed in other studies $[18,19]$. American studies showed a change between lung cancer and education from a positive to a negative association around 1970 among men and mid-1980s for women. For both men and women inequalities increased after the change [18, 
19]. This reversal occurred earlier in the US due to an advanced stage of the smoking epidemic there. However, the American study did not report a quicker widening of socioeconomic inequalities in lung cancer mortality among women than among men after this reversal took place.

Smoking and alcohol are well known risk factors for UADT cancer, with a larger population attributable risk (PAR) for smoking than for alcohol [20]. Inequalities in UADT cancer mortality are then partly explained by inequalities in smoking and alcohol consumption [21]. The large decrease in UADT cancer mortality among men can be explained by a decrease in smoking rates and alcohol consumption in France during the last decades $[22,23]$. In particular, a large decrease (approximately 30\%) in alcohol consumption has been observed from 1960 to 2001 [23]. We observed that despite of the decrease in mortality rates, inequalities remained stable over time. Literature on socioeconomic differences in alcohol consumption is sparse. However one study reported that around 1990 in France inequalities were found in excessive alcohol consumption [24]. Our results suggest that alcohol consumption strongly decreased in all educational groups but that relative inequalities in alcohol consumption are very likely to have remained over time.

A major strength of this study is that it is based on a large sample representative of the French population. Our study might be limited by the fact that education as indicator for socioeconomic status is debatable. Advantages are that it is stable over life course and shown to be a good predictor of mortality; a higher education facilitates a lifelong choice for a healthy behavior and provides opportunities for choosing a working environment that result in a certain level of income, working and living conditions and accessibility of healthcare [2527]. However it is fixed early in adult life and therefore later socio economic changes are not 
taken into account [27]. This could have influenced our results in underestimating the social inequalities for the older people in our study population.

The percentage of missing values on education differs between the two periods due to some internal procedures performed by Insee on the 1990 census dataset. This could have slightly altered the results; however we expect the influence, if any, to be small.

In addition there have been considerable changes in educational opportunities for women [28], as our results showed the educational level is increasing among women and especially among younger women. To account for this the RII was used as it takes into account the size and the relative position of the different educational groups.

This study shows the magnitude of educational differences in lung and UADT cancer mortality in France during the last two decades. UADT cancer mortality rates among men decreased strongly for every educational level. This implies that, although relative inequalities remained stable in UADT cancer mortality over time, the population health burden that is associated with social inequalities in UADT cancer mortality decreased substantially. Inequalities in lung cancer mortality are increasing. Widening inequalities among the younger men implicate that the decrease in mortality rates is more in favor of the highly educated men. Based on the smoking epidemic and trends in the US we expect the inequalities to widen even more. The trends for women in lung cancer are concerning. We expect an increase both in the lung cancer mortality rates and educational differences among women in the future. Moreover differences in the magnitude of inequalities may disappear between men and women in the coming decades. Consequently, tobacco control policies should really ensure they reach the men and women with a lower socioeconomic position.

\section{Acknowledgements}


This study is funded by the French National Cancer Institute (INCa), grant no R06074LL. 


\section{References}

1. Ferlay J, Parkin DM, and Steliarova-Foucher E. Estimates of cancer incidence and mortality in Europe in 2008. Eur J Cancer, 2010; 46(4):765-81.

2. Bray FI and Weiderpass E. Lung cancer mortality trends in 36 European countries: secular trends and birth cohort patterns by sex and region 1970-2007. Int J Cancer, 2010; 126(6):1454-66.

3. Levi F, Bosetti C, Fernandez E, Hill C, Lucchini F, Negri E, et al. Trends in lung cancer among young European women: the rising epidemic in France and Spain. Int $J$ Cancer, 2007; 121(2):462-5.

4. Yako-Suketomo H and Matsuda T. Comparison of time trends in lip, oral cavity and pharynx cancer mortality (1990-2006) between countries based on the WHO mortality database. Jpn J Clin Oncol, 2010; 40(11):1118-9.

5. Mackenbach JP, Huisman M, Andersen O, Bopp M, Borgan JK, Borrell C, et al. Inequalities in lung cancer mortality by the educational level in 10 European populations. Eur J Cancer, 2004; 40(1):126-35.

6. Van der Heyden JH, Schaap MM, Kunst AE, Esnaola S, Borrell C, Cox B, et al. Socioeconomic inequalities in lung cancer mortality in 16 European populations. Lung Cancer, 2009; 63(3):322-30.

7. Menvielle G, Kunst AE, Stirbu I, Strand BH, Borrell C, Regidor E, et al. Educational differences in cancer mortality among women and men: a gender pattern that differs across Europe. Br J Cancer, 2008; 98(5):1012-9.

8. Borrell C, Azlor E, Rodriguez-Sanz M, Puigpinos R, Cano-Serral G, Pasarin MI, et al. Trends in socioeconomic mortality inequalities in a southern European urban setting at the turn of the 21st century. J Epidemiol Community Health, 2008; 62(3):258-66.

9. Menvielle G, Leclerc A, Chastang JF, Melchior M, and Luce D. Changes in socioeconomic inequalities in cancer mortality rates among French men between 1968 and 1996. Am J Public Health, 2007; 97(11):2082-7.

10. Anderson RN, Minino AM, Hoyert DL, and Rosenberg HM. Comparability of cause of death between ICD-9 and ICD-10: preliminary estimates. Natl Vital Stat Rep, 2001; 49(2):1-32.

11. Mackenbach JP and Kunst AE. Measuring the magnitude of socio-economic inequalities in health: an overview of available measures illustrated with two examples from Europe. Soc Sci Med, 1997; 44(6):757-71.

12. Regidor E. Measures of health inequalities: part 2. J Epidemiol Community Health, 2004; 58(11):900-3.

13. Hart CL, Hole DJ, Gillis CR, Smith GD, Watt GC, and Hawthorne VM. Social class differences in lung cancer mortality: risk factor explanations using two Scottish cohort studies. Int J Epidemiol, 2001; 30(2):268-74.

14. Menvielle G, Boshuizen H, Kunst AE, Dalton SO, Vineis P, Bergmann MM, et al. The role of smoking and diet in explaining educational inequalities in lung cancer incidence. J Natl Cancer Inst, 2009; 101(5):321-30.

15. Lopez AD, Colloshaw NE, and Piha T. A descriptive model of the cigarette epidemic in developed countries. Tobacco Control, 1994; 3:242-247.

16. Aliaga C. Tobacco: twenty years of use and consumption [article in French] Insee première, 2001.

17. Cavelaars AEJM, Kunst, A E, Geurts, J J M , Crialesi, R, Grötvedt L, Helmert U , Lahelma E, Lundberg O, Matheson, J, Mielck, A,Rasmussen, N Kr , Regidor, E, do 
RosárioGiraldes, M, Spuhler, Th,, and Mackenbach JP. Educational differences in smoking: international comparison. BMJ, 2000; 320:1102-1107.

18. Singh GK, Miller BA, and Hankey BF. Changing area socioeconomic patterns in U.S. cancer mortality, 1950-1998: Part II--Lung and colorectal cancers. J Natl Cancer Inst, 2002; 94(12):916-25.

19. Kinsey T, Jemal A, Liff J, Ward E, and Thun M. Secular trends in mortality from common cancers in the United States by educational attainment, 1993-2001. J Natl Cancer Inst, 2008; 100(14):1003-12.

20. Hashibe M, Brennan P, Chuang SC, Boccia S, Castellsague X, Chen C, et al. Interaction between tobacco and alcohol use and the risk of head and neck cancer: pooled analysis in the International Head and Neck Cancer Epidemiology Consortium. Cancer Epidemiol Biomarkers Prev, 2009; 18(2):541-50.

21. Menvielle G, Luce D, Goldberg P, and Leclerc A. Smoking, alcohol drinking, occupational exposures and social inequalities in hypopharyngeal and laryngeal cancer. Int J Epidemiol, 2004; 33(4):799-806.

22. Hill C. Trends in tobacco smoking and consequences on health in France. Prev Med, 1998; 27(4):514-9.

23. Besson D. Alcoholic beverages: 40 years of decline in consumption [article in French] Insee Première, 2004.

24. Cavelaars AEJM, Kunst, A.E., Mackenbach, J.P. Socio-economic differences in risk factors for morbidity and mortality in European Community: an international comparison. Journal of Health Psychology, 1997; 2(3):353-372.

25. Geyer S, Hemstrom O, Peter R, and Vagero D. Education, income, and occupational class cannot be used interchangeably in social epidemiology. Empirical evidence against a common practice. J Epidemiol Community Health, 2006; 60(9):804-10.

26. Davey Smith G, Hart C, Hole D, MacKinnon P, Gillis C, Watt G, et al. Education and occupational social class: which is the more important indicator of mortality risk? $J$ Epidemiol Community Health, 1998; 52(3):153-60.

27. Berkman LF and Macintyre S. The measurement of social class in health studies: old measures and new formulations. IARC Sci Publ, 1997(138):51-64.

28. Galobardes B, Shaw M, Lawlor DA, Lynch JW, and Davey Smith G. Indicators of socioeconomic position (part 2). J Epidemiol Community Health, 2006; 60(2):95-101. 